\title{
Characterization of the Long-Term Interaction between Intracellular Reactive Oxygen Species and Oxidative DNA Damage in Murine Lin-/Sca-1+ Cells Exposed to Ionizing Radiation
}

\author{
Junya Ishikawa ${ }^{1,2 *}$, Taro Morisaki² \\ ${ }^{1}$ Department of Medical Radiologic Technology, Kyorin University, Mitaka, Japan \\ ${ }^{2}$ Department of Environmental Health Science, Oita University of Nursing and Health Sciences, Oita, Japan \\ Email: ‘ishikawaj@ks.kyorin-u.ac.jp
}

How to cite this paper: Ishikawa, J. and Morisaki, T. (2019) Characterization of the Long-Term Interaction between Intracellular Reactive Oxygen Species and Oxidative DNA Damage in Murine Lin $^{-} / \mathrm{Sca}-1^{+}$Cells Exposed to Ionizing Radiation. International Journal of Medical Physics, Clinical Engineering and Radiation Oncology, 8, 95-105.

https://doi.org/10.4236/ijmpcero.2019.82009

Received: March 26, 2019

Accepted: May 17, 2019

Published: May 20, 2019

Copyright () 2019 by author(s) and Scientific Research Publishing Inc. This work is licensed under the Creative Commons Attribution International License (CC BY 4.0).

http://creativecommons.org/licenses/by/4.0/

(c) $\underset{\mathrm{EY}}{\mathrm{B}}$ Open Access

\begin{abstract}
Mutations in the Sfpil gene are essential for the development of radiation-induced acute myeloid leukemia. In this study, we investigated long-term interaction among immature hematopoietic cell number, intracellular reactive oxygen species contents, and oxidative DNA damage frequency after irradiation. $\mathrm{Lin}^{-} / \mathrm{Sca}-1^{+}$cells were isolated from $\mathrm{C} 3 \mathrm{H} / \mathrm{HeN}$ mice on days $1-400$ after 0 - 3 Gy total body irradiation. On days $1-7$, the number of surviving cells decreased and reached a minimum; however, the number of cells gradually recovered until day 200 . Intracellular reactive oxygen species contents significantly increased from day 1 to day 30 . In addition, the frequency of oxidative DNA damage tended to increase from day 1 and day 30 , and that at day 30 was significantly increased in the 3 Gy group compared with that in the control group. In contrast, decreased cell number, increased intracellular reactive oxygen species content, and decreased oxidative DNA damage frequency were observed on day 400 . These results suggested that oxidative DNA damage was involved in intracellular reactive oxygen species generation induced by cell proliferation to compensate for cell death after irradiation.
\end{abstract}

\section{Keywords}

Acute Myeloid Leukemia, Oxidative DNA Damage, Reactive Oxygen Species

\section{Introduction}

Ionizing radiation has many beneficial applications, including uses in medicine, industry, and research. Exposure to ionizing radiation causes damage to DNA, 
proteins, and lipids in mammalian cells induces mitochondria-dependent generation of reactive oxygen species (ROS), with subsequent induction of cell cycle arrest, apoptosis, and stress-related responses, including alterations to gene expression [1] [2] [3]. Radiation contributes to carcinogenesis via a multistage process [4] [5] and/or genomic instability [6] [7]; however, the exact mechanisms are still unclear. Because beneficial applications of radiation are indispensable, elucidation of the mechanisms of radiation-induced carcinogenesis is a socially important issue.

Acute myeloid leukemia (AML) is a representative human disease induced by radiation exposure. Many previous studies have used mouse models to study radiation-induced AML [8]-[19]. These reports have demonstrated that a latency period of more than 1 - 2 years is required [8] [9] [10] [11] [12] and that a deletion of chromosome 2 [13] [14] [15] [16] and a mutation in the Sfpil gene, also known as the $P U .1$ gene, on the retained homolog [17] [18] [19] are essential for the pathogenesis of radiation-induced AML. Moreover, radiation-induced deletion is known to occur early, i.e. within $24 \mathrm{~h}$ after irradiation [14] [20]. Ban and Kai proposed a mechanism for the pathogenesis of radiation-induced AML using a mathematical model [21]. They suggested that this process may be divided into three steps, as follows: 1) irradiation immediately induces cell death and deletion of chromosome 2; 2) cell proliferation to compensate for dead cells induces hematopoietic stem cell senescence caused by replicative stress; and 3) AML develops because of increased intracellular ROS generation accompanied by cell senescence, resulting in spontaneous point mutations [21]. The Sfpil mutation found in radiation-induced AML is typically a point mutation at codon 235 of the DNA-binding Ets domain, particularly a C:G to T:A transition at the CpG site [17] [18] [19] [21], which is the most common spontaneous mutation induced by intracellular ROS [21] [22] [23]. However, the detailed mechanisms mediating mutations in the Sfpil gene, which is an essential factor affecting the development of radiation-induced AML, remain unclear.

Accordingly, we attempted to experimentally verify the mathematical model proposed by Ban and Kai, as a part of elucidating pathogenesis of radiation-induced AML. Practically, in this study, we investigated the long-term interactions among the number of immature hematopoietic cells, levels of intracellular ROS, and frequency of oxidative DNA damage after irradiation in order to elucidate the detailed mechanisms mediating the development of radiation-induced AML.

\section{Materials and Methods}

\subsection{Animals and Gamma-Irradiation}

This study was approved by the Committee of Research Ethics and Safety in Oita University of Nursing and Health Sciences (Oita, Japan). All experiments were conducted according to legal regulations in Japan and following the Guideline for Animal Experiments of Oita University of Nursing and Health Sciences. 
Male $\mathrm{C} 3 \mathrm{H} / \mathrm{HeN}$ Jcl mice, as a model mouse for AML, were delivered at 7 weeks of age from the breeding facility of Clea Japan (Tokyo, Japan). At 8 weeks of age, five mice in each group were exposed to 0,1 , and $3 \mathrm{~Gy}$ of ${ }^{137} \mathrm{Cs}$ gamma-rays at a dose rate of $0.72 \mathrm{~Gy} / \mathrm{min}$ by Gammacell 40 Exactor (Marubeni Utility Service, Tokyo, Japan). All mice were housed in a temperature-controlled room under a 12 -h light/dark cycle in a conventional animal breeding facility. The mice received food and water ad libitum.

\subsection{Collection and Purification of Lin-/Sca-1+ Cells as Hematopoietic Stem/Progenitor Cells}

Mice were sacrificed under deep anesthesia at $0,1,7,30,90,200$, and 400 days after exposure to 0,1 , and $3 \mathrm{~Gy}$ total body irradiation (105 mice in total). Briefly, mononuclear cells were isolated from the bilateral femur by flushing with phosphate-buffered saline (PBS). The cells were then processed for lineage-negative $\left(\mathrm{Lin}^{-}\right)$cell enrichment according to the manufacturer's instructions. An EasySep Mouse Hematopoietic Progenitor Cell Enrichment Kit (Veritas Corporation, Tokyo, Japan) was used for the negative selection of Lin cells. The cells were then processed for Sca- $1^{+}$cell enrichment according to the manufacturer's instructions. An EasySep Mouse SCA1 Positive Selection Kit (Veritas Corporation) was used for the positive selection of Sca- $1^{+}$cells. Using the procedure described above, $\mathrm{Lin}^{-} / \mathrm{Sca}-1^{+}$cells were obtained; although this population is heterogeneous, this isolated $\mathrm{Lin}^{-} / \mathrm{Sca}-1^{+}$-enriched cell population was composed of hematopoietic stem and progenitor cells.

\subsection{Number of Survival Cells}

After isolation of $\mathrm{Lin}^{-} / \mathrm{Sca}-1^{+}$cells, some of the cells for each condition were harvested, and the viable cells were stained and counted using Burker-Turk-solution (Nacalai Tesque, Kyoto, Japan) under a microscope. The relative value normalized to the control value was calculated as the ratio of the number of gamma-irradiated cells to the number of non-irradiated cells at the same time point.

\subsection{Intracellular ROS Contents}

The intracellular ROS contents in $\mathrm{Lin}^{-} / \mathrm{Sca}-1^{+}$cells were analyzed using CellROX Green Oxidative Stress Reagents (Life Technologies, Carlsbad, CA, USA) with a Tali Image-Based Cytometer (Life Technologies). Briefly, after irradiation and breeding for the indicated period, the mice were sacrificed, and $\mathrm{Lin}^{-} / \mathrm{Sca}-1^{+}$cells were isolated from the femur. These cells were stained with $5 \mu \mathrm{M}$ CellROX Green Oxidative Stress Reagent for $30 \mathrm{~min}$ at $37^{\circ} \mathrm{C}$ in the dark. After labeling, unincorporated CellROX Green Oxidative Stress Reagent was removed by three washes with PBS. Each sample was resuspended in PBS and analyzed using an image-based cytometer. For each experiment, unstained cells were used as a negative control. 


\subsection{Total DNA Extraction}

Total DNA was extracted from $\mathrm{Lin}^{-} / \mathrm{Sca}-1^{+}$cells (five samples for each dose). Total DNA was extracted using a DNeasy Blood and Tissue Kit (Qiagen, Bothell, WA, USA) according to the manufacturer's instructions, and the DNA concentration was determined using a fluorometer (Qubit 3.0 Fluorometer; Life Technologies) and Qubit dsDNA HS Assay Kit (Life Technologies).

\subsection{Oxidative DNA Damage}

Oxidative DNA damage was investigated using an Oxiselect Oxidative DNA Damage ELISA Kit (Cell Biolabs, Inc., San Diego, CA, USA) according to the manufacturer's instructions. This kit contained a competitive enzyme-linked immunosorbent assay (ELISA) for the quantitative measurement of 8-hydroxyguanosine (8-OHdG). Briefly, after irradiation and breeding for the indicated period, the mice were sacrificed, and $\mathrm{Lin}^{-} / \mathrm{Sca}-1^{+}$cells were isolated from the femur. Total DNA extracted from these cells was digested, and the mixture was centrifuged. The supernatant was collected and applied for the ELISA. Five microliters of the supernatant or standard were added to each well of 8-OHdG conjugate-coated plates. The plate was incubated for $10 \mathrm{~min}$ at room temperature on an orbital shaker. After incubation, diluted anti-8-OHdG antibodies were added ( $5 \mu \mathrm{L} /$ well), and plates were incubated for $60 \mathrm{~min}$ at room temperature on an orbital shaker. All wells were then washed and stripped three times, and excess wash buffer was completely removed. Next, diluted secondary antibody-enzyme conjugate was added $(100 \mu \mathrm{L} /$ well), and plates were incubated for $60 \mathrm{~min}$ at room temperature on an orbital shaker. After incubation, all wells were washed and stripped four times. Subsequently, substrate solution was added $(100 \mu \mathrm{L} /$ well $)$, and plates were incubated for $5 \mathrm{~min}$ at room temperature. Then, stop solution was added $(100 \mu \mathrm{L} /$ well). Finally, the absorbance of each well was read using a microplate reader (Tecan Japan Co., Ltd., Kanagawa, Japan) at $450 \mathrm{~nm}$.

\subsection{Statistical Analysis}

The differences among the non-irradiated and irradiated groups were analyzed by multiple comparison tests, such as Steel's test. Statistical analysis was performed using Excel 2007 (Microsoft, WA, USA) with the add-in software Statcel 3 (OMS, Saitama, Japan). Statistically significant differences were defined at a significance level of $5 \%$.

\section{Results}

\subsection{Number of Surviving Lin-/Sca-1+ Cells Exposed to Gamma-Irradiation}

First, we examined cell survival/death of $\mathrm{Lin}^{-} / \mathrm{Sca}-1^{+}$cells isolated from the bilateral femurs of mice after 1 or $3 \mathrm{~Gy}$ total body gamma-irradiation. The results showed that the number of viable cells was immediately decreased after irradiation with 1 or $3 \mathrm{~Gy}$ and reached a minimum value at day 3 or day 1 after irradia- 
tion, respectively (Figure 1), with viable gamma-irradiated cells decreasing to $73 \% \pm 35 \%$ or $27 \% \pm 5 \%$ that of control non-irradiated cells, respectively. Subsequently, the number of viable cells gradually increased, returning to the value of the control at day 200 after irradiation.

However, cells exposed to 3 Gy significantly decreased again by about $50 \%$ compared with non-irradiated cells on day 400 after irradiation.

\subsection{Intracellular ROS Contents in Surviving Lin-/Sca-1+Cells Exposed to Gamma-Irradiation}

Intracellular ROS contents were significantly elevated, reaching a maximum value in $\mathrm{Lin}^{-} / \mathrm{Sca}-1^{+}$cells on day 1 after gamma-irradiation of about twice that of control cells (Figure 2). Although this significant increase in ROS was observed until day 7 , intracellular ROS levels were significantly decreased on day 90 , when the surviving fraction equalized with that of the non-irradiated control in the 3 Gy-irradiated group. In contrast, intracellular ROS was significantly increased on day 30 in the 1 Gy-irradiated group. The generation of ROS gradually decreased to the level of the non-irradiated control by day 200 and significantly increased again on day 400 after irradiation in irradiated $\mathrm{Lin}^{-} / \mathrm{Sca}-1^{+}$cells.

\subsection{Detection of Oxidative DNA Damage in Surviving Lin-/Sca-1+ Cells Exposed to Gamma-Irradiation}

DNA is the most biologically significant target of oxidative attack, and continuous oxidative damage to DNA is a significant contributor to the age-related development of several cancers. Among numerous types of oxidative DNA damage, the formation of $8-\mathrm{OHdG}$ is a ubiquitous marker of oxidative stress targeting DNA [24]. Therefore, we next analyzed the levels of oxidative DNA damage in $\mathrm{Lin}^{-} / \mathrm{Sca}-1^{+}$cells isolated from the bilateral femurs of mice after 1 or 3 Gy total body gamma-irradiation (Figure 3).

Compared with non-irradiated cells, the relative fluorescence intensity of 8-OHdG tended to increase in irradiated $\mathrm{Lin}^{-} / \mathrm{Sca}-1^{+}$cells on day 1 after gamma-irradiation, although this difference was not statistically significant. Subsequently, 8-OHdG levels were elevated again in both irradiated groups on day 30 after irradiation, particularly in the 3 Gy group. Importantly, this elevation was observed at the time point of cell survival recovery to the control level. In contrast, 8-OHdG levels were significantly decreased in the 3 Gy group on day 400 after irradiation.

\section{Discussion}

In the present study, we aimed to elucidate the detailed mechanisms mediating mutations including Sfpil gene, which are essential for the development of radiation-induced AML, by investigating the long-term interactions among the number of immature hematopoietic cells, generation of intracellular ROS, and frequency of oxidative DNA damage after irradiation. Gamma-irradiation decreased 


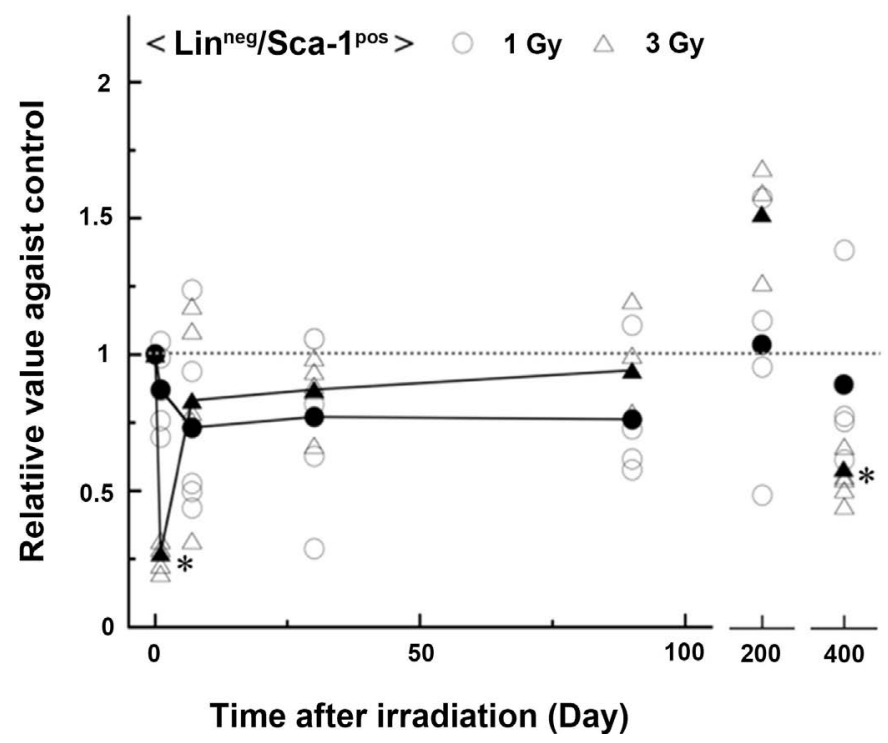

Figure 1. Number of survival $\mathrm{Lin}^{-} / \mathrm{Sca}-1^{+}$cells after gamma-irradiation. Viable cells were stained using Burker-Turk-solution and counted under a microscope. The relative value normalized to the control value was calculated as the ratio of the number of gamma-irradiated cells to the number of non-irradiated cells at the same time. The filled symbols show means, and unfilled symbols show the values of individual samples. ${ }^{\star}$ Statistically significant differences, defined as a significance level of $5 \%$, by Steel's test.

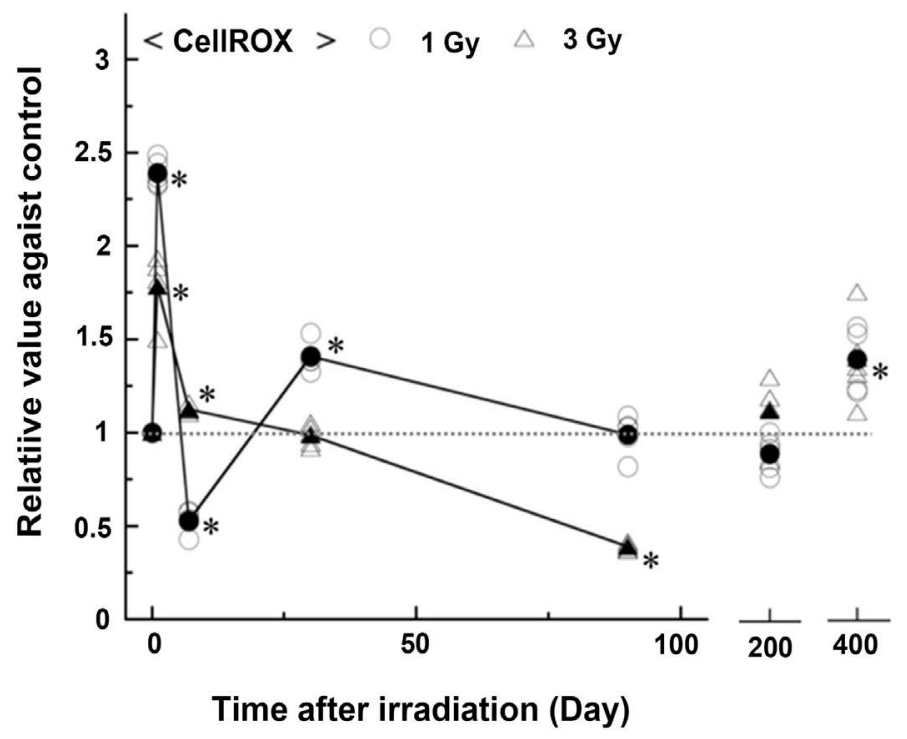

Figure 2. Intracellular ROS contents in $\mathrm{Lin}^{-} / \mathrm{Sca}-1^{+}$cells after gamma-irradiation. Detection of intracellular ROS in the $\mathrm{Lin}^{-} / \mathrm{Sca}-1^{+}$cells was performed using CellROX Green Oxidative Stress Reagent and an image-based cytometer. The relative value normalized to the control value was calculated as the ratio of the fluorescence intensity of gamma-irradiated cells to that of non-irradiated cells at the same time. The filled symbols show means, and unfilled symbols show the values of individual samples. ${ }^{\star}$ Statistically significant differences, defined as a significance level of $5 \%$, by Steel's test. 


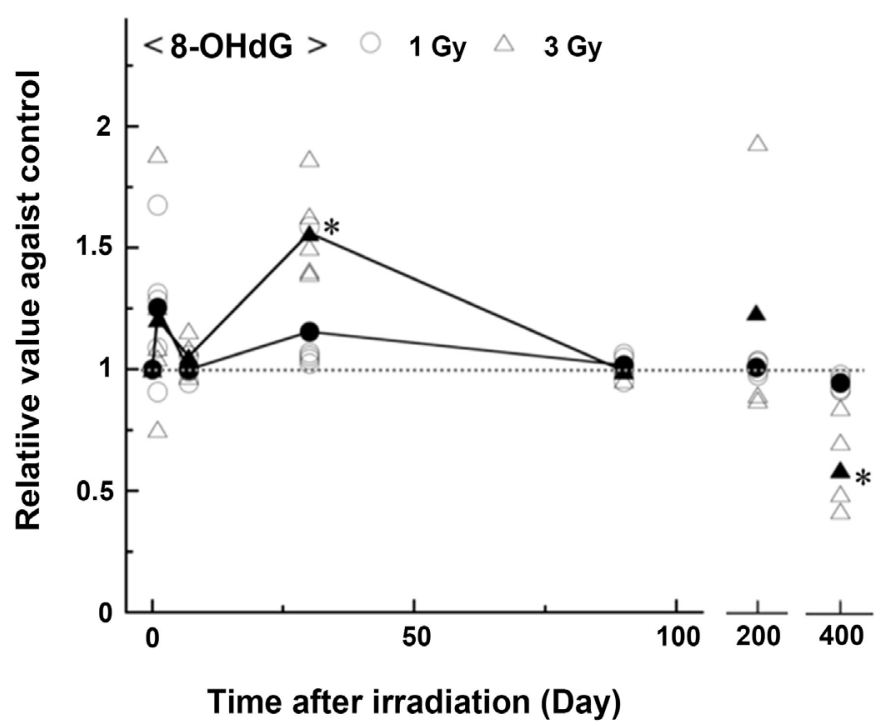

Figure 3. Frequency of oxidative DNA damage in $\mathrm{Lin}^{-} / \mathrm{Sca}-1^{+}$cells after gamma-irradiation. Detection of $8-\mathrm{OHdG}$, as an indicator of oxidative DNA damage, was performed in $\mathrm{Lin}^{-} / \mathrm{Sca}-1^{+}$cells using ELISA. The concentration of 8 -OHdG was calculated as the ratio of the absorbance of gamma-irradiated DNA extracts to that of the established standard. The relative value normalized to the control value was then calculated as the ratio of the 8-OHdG concentration in gamma-irradiated samples relative to that in nonirradiated samples at the same time. The filled symbols show means, and unfilled symbols show the values of individual samples. The values show five separate experiments performed in triplicate wells. *Statistically significant differences, defined as a significance level of $5 \%$, by Steel's test.

the number of $\mathrm{Lin}^{-} / \mathrm{Sca}-1^{+}$cells isolated from $\mathrm{C} 3 \mathrm{H} / \mathrm{HeN}$ mice on day 1 in the 3 Gy group and day 7 in the 1 Gy group. Furthermore, the number of surviving cells gradually recovered until days 90 and 200, respectively, and intracellular ROS contents significantly increased from day 1 to day 30 in both irradiated groups. In addition, the frequency of oxidative DNA damage tended to increase in both irradiated groups on days 1 and 30. However, on day 400, a significant decrease in the number of cells, significant increase in intracellular ROS contents, and significant decrease in the frequency of oxidative DNA damage were observed in the 3 Gy group, and similar trends were observed in the 1 Gy group. These findings provided important insights into the effects of gamma-irradiation on carcinogenesis, particularly AML.

Few researchers have investigated the long-term effects of radiation on hematopoietic stem/progenitor cells. However, several short- and/or moderate-term studies have been published, with study periods of up to 60 days. Wang et al. reported that $\mathrm{Lin}^{-} / \mathrm{Sca}-1^{+} / \mathrm{c}-\mathrm{Kit}^{+}$cells exhibited an immediate transient decrease after irradiation and then gradually recovered via proliferation to compensate for the observed cell death [25]. Additionally, they demonstrated that the multipotency and self-renewal abilities of $\mathrm{Lin}^{-} / \mathrm{Sca}-1^{+} / \mathrm{c}-\mathrm{Kit}^{+}$cells were both attenuated by proliferation after irradiation [25] [26]. Moreover, persistent intracel- 
lular ROS induced by radiation resulted in premature senescence of hematopoietic cells [25] [26] [27] [28]. Additionally, increased intracellular ROS contents and/or premature senescence of hematopoietic cells resulted in radiation-induced residual bone marrow suppression [26] [29] [30]. These results suggested that to compensate for radiation-induced cell death, proliferation accompanied by increased intracellular ROS accelerated the premature senescence of immature hematopoietic cells and resulted in radiation-induced residual bone marrow suppression. Our results indicated that compensation for transient radiation-induced cell death via cell proliferation resulted in intermittent increases in intracellular ROS until day 200, consistent with previous reports.

Previous studies have shown that mutations in the Sfpil gene found in radiation-induced AML are primarily C:G to T:A transition mutations [17] [18] [19] [21], which are the most common type of spontaneous mutation induced by intracellular ROS [21] [22] [23]. The results of a mathematical model developed by Ban and Kai supported these findings [21]. In addition, Chung et al. demonstrated that the mutation frequency increased significantly by exposure to oxidative stress [31]. Therefore, these findings indicated that spontaneous ROS caused point mutations, including mutations in Sfpi1. In fact, Wang et al. and Phazhanisamy et al. reported that ionizing radiation significantly increased oxidative DNA stress on day 30 [2] [32], supporting our current results. Indeed, our findings suggested that transitions promoting the development of radiation-induced AML occurred on days 1 and 30, when increased DNA damage and intracellular ROS contents were observed. Therefore, we concluded that the period from days 1 to 30 was important for clarifying the mechanisms mediating the development of radiation-induced AML. Further studies are needed to fully elucidate these mechanisms.

In contrast, our results revealed that gamma-irradiation decreased the number of cells, increased intracellular ROS contents, and decreased the frequency of 8-OHdG-positive cells on day 400 in both groups. Although we assumed that oxidative DNA damage would accumulate in mice with radiation-induced AML, these results were contrary to our expectations. Previous reports have demonstrated that ionizing radiation accelerates cell senescence and results in residual bone marrow suppression [25] [26] [27] [28] [29]. Additionally, the frequency of DNA double-strand breaks (DSBs) related to accumulation of ROS was increased in senescent cells [26]. Indeed, our colleagues investigated the frequency of DSBs in mouse bone marrow cells on day 400 using 53BP1 immunofluorescence staining and found a 1.3-fold increase in the 1 Gy group and a 1.2-fold increase in the $3 \mathrm{~Gy}$ group compared with that in the non-irradiated control (data not shown). These results suggested that cell senescence related to persistent ROS decreased the number of immature hematopoietic cells by increasing the frequency of DSBs. Moehrle et al. demonstrated that hematopoietic stem cells acquired apoptosis in order to recover from point mutations caused by DNA damage [33]. Accordingly, immature hematopoietic cells may have been exhausted after irradiation in mice that did not develop radiation-induced AML, 
and immature hematopoietic cells containing mutations including 8-OHdG may have been relatively easy to eliminate after irradiation. However, further studies are required to verify these assertions.

In conclusion, our current findings suggested that oxidative DNA damage, which is essential for the pathogenesis of radiation-induced AML, was involved in intracellular ROS generated by cell proliferation to compensate for cell death. However, this study was performed under restricted conditions, such as only 1 and $3 \mathrm{~Gy}$ of $\mathrm{X}$-irradiation and five samples in each group. Also, to minimize the number of animals in this study, we cannot use a large number of animals resulting none of the mice actually developed radiation-induced AML, and further studies with more mice are needed to obtain AML model mice. Additionally, these limitations may be overcome through development of mathematical models and/or novel experimental methods. In any case, our findings provide important insights into the mechanisms of radiation-induced AML and with being valuable for later more detailed studies.

\section{Acknowledgements}

This work was supported by a Grant-in-Aid for Encouragement of Scientists from Oita University of Nursing and Health Sciences (2014-2015).

\section{Conflicts of Interest}

The authors declare no conflicts of interest regarding the publication of this paper.

\section{References}

[1] Ishikawa, J., Hayashi, N., Yamaguchi, M., Monzen, M. and Kashiwakura, I. (2015) Characteristics of Human CD34 ${ }^{+}$Cells Exposed to Ionizing Radiation under Cytokine-Free Condition. Journal of Radiation Research, 56, 678-690. https://doi.org/10.1093/jrr/rrv024

[2] Kaneyuki, Y., Yoshino, H. and Kashiwakura, I. (2012) Involvement of Intracellular Reactive Oxygen Species and Mitochondria in the Radiosensitivity of Human Hematopoietic Stem Cells. Journal of Radiation Research, 53, 145-150. https://doi.org/10.1269/jrr.11099

[3] Kato, K., Takahashi, K., Monzen, S., Yamamoto, H., Maruyama, A., Itoh, K. and Kashiwakura, I. (2010) Relationship between Radiosensitivity and Nrf2 Target Gene Expression in Human Hematopoietic Stem Cells. Radiation Research, 174, 177-184. https://doi.org/10.1667/RR2146.1

[4] Vogelstein, B., Fearon, R., Hamilton, S.R., Kem, S.E., Preisinger, A.C., Leppert, M., Nakamura, Y., White, R., Smits, A.M. and Bos, J.K. (1988) Genetic Alterations during Colorectal-Tumor Development. The New England Journal of Medicine, 319, 525-532. https://doi.org/10.1056/NEJM198809013190901

[5] Fearon, E.R. and Volgelstein, B. (1990) A Genetic Model for Colorectal Tumorigenesis. Cell, 61, 759-767. https://doi.org/10.1016/0092-8674(90)90186-I

[6] Little, J.B. (2000) Radiation Carcinogenesis. Carcinogenesis, 21, 397-404. https://doi.org/10.1093/carcin/21.3.397

[7] Lengauer, C., Kinzler, K.W. and Vogelstein, B. (1998) Genetic Instabilities in Hu- 
man Cancers. Nature, 396, 643-649. https://doi.org/10.1038/25292

[8] Upton, A.C., Wolff, F.F., Furth, J. and Kimball, A.W. (1958) A Comparison of the Induction of Myeloid and Lymphoid Leukemias in X-Irradiated RF Mice. Cancer Research, 18, 842-848.

[9] Major, I.R. (1979) Induction of Myeloid Leukemia by Whole-Body Single Exposure of CBA Male Mice to X-Rays. British Journal of Cancer, 40, 903-913. https://doi.org/10.1038/bjc.1979.284

[10] Mole, R.H., Papworth, D.G. and Corp, M.J. (1983) The Dose-Response for X-Ray Induction of Myeloid Myeloid Leukemia in Male CBA/H Mice. British Journal of Cancer, 47, 285-291. https://doi.org/10.1038/bjc.1983.37

[11] Resnitzky, P., Estrov, Z. and Haran-Ghera, N. (1985) High Incidence of Acute Myeloid Leukemia in SJL/J Mice after X-Irradiation and Corticosteroid. Leukemia Research, 9, 1519-1528. https://doi.org/10.1016/0145-2126(85)90045-1

[12] Seki, M., Yoshida, K., Nishimura, M. and Nemoto, K. (1991) Radiation-Induced Myeloid Leukemia in $\mathrm{C} 3 \mathrm{H} / \mathrm{He}$ Mice and the Effect of Prednisolone Acetate on Leukemogenesis. Radiation Research, 127, 146-149.

https://doi.org/10.2307/3577958

[13] Hayata, I., Seki, M., Yoshida, K., Hirashima, K., Sado, T., Yamagiwa, J. and Ishihara, T. (1983) Chromosomal Aberrations Observed in 52 Mouse Myeloid Leukemias. Cancer Research, 43, 367-373.

[14] Trakhtenbrot, L., Krauthgamer, R., Resnitzky, P. and Haran-Ghera, N. (1988) Deletion of Chromosome 2 Is an Early in the Development of Radiation-Induced Myeloid Leukemia in SJL/J Mice. Leukemia, 2, 545-550.

[15] Rithidech, K.N., Bond, V.P., Cronkite, E.P. and Thompson, M.H. (1993) A Specific Chromosomal Deletion in Murine Leukemic Cells Induced by Radiation with Different Qualities. Experimental Hematology, 21, 427-431.

[16] Clark, D.J., Meijne, E.I., Bouffler, S.D., Huiskamp, R., Skidmore, C.J., Cox, R. and Silver, A.R. (1996) Microsatellite Analysis of Recurrent Chromosome 2 Deletions in Acute Myeloid Leukemia Induced by Radiation in F1 Hybrid Mice. Genes, Chromosomes and Cancer, 16, 238-246. https://doi.org/10.1002/(SICI)1098-2264(199608)16:4<238::AID-GCC3>3.0.CO;2-Z

[17] Cook, W.D., McCaw, B.J., Herring, C., John, D.L., Foote, S.J., Nutt, S.L., Adams, J.M. (2004) PU.1 Is a Suppressor of Myeloid Leukemia, Inactivated in Mice by Gene Deletion and Mutation of Its DNA Binding Domain. Blood, 104, 3437-3444. https://doi.org/10.1182/blood-2004-06-2234

[18] Suraweera, N., Meijne, E., Moody, J., Carrvajal-Carmona, L.G., Yoshida, K., Pollard, P., Fitzgibbon, J., Riches, A., van Laar, T., Huiskamp, R., Rowan, A., Tomlinson, I.P. and Silver, A. (2005) Mutations of the PU.1 Ets Domain Are Specifically Associated with Murine Radiation-Induced, but Not Human Therapy-Related, Acute Myeloid Leukaemia. Oncogene, 24, 3678-3683. https://doi.org/10.1038/sj.onc.1208422

[19] Hirouchi, T., Takabatake, T., Yoshida, K., Nitta, Y., Nakamura, M., Tanaka, S., Ichinose, K., Oghiso, Y. and Tanaka, K. (2008) Upregulation of c-myc Gene Accompanied by PU.1 Deficiency in Radiation-Induced Acute Myeloid Leukemia in Mice. Experimental Hematology, 36, 871-885. https://doi.org/10.1016/j.exphem.2008.01.015

[20] Ban, N., Kai, M. and Kusama, T. (1997) Chromosome Aberrations in Bone Marrow Cells of C3/He Mice at an Early Stage after Whole-Body Irradiation. Journal of Radiation Research, 38, 219-231. https://doi.org/10.1269/jrr.38.219

[21] Ban, N. and Kai, M. (2009) Implication of Replicative Stress-Related Stem Cell 
Ageing in Radiation-Induced Murine Leukaemia. British Journal of Cancer, 101, 363-371. https://doi.org/10.1038/sj.bjc.6605135

[22] Douglas, G.R., Gingerich, J.D., Gossen, J.A. and Bartlett, S.A. (1994) Sequence Spectra of Spontaneous lacZ Gene Mutations in Transgenic Mouse Somatic and Germline Tissues. Mutagenesis, 9, 451-458. https://doi.org/10.1093/mutage/9.5.451

[23] Ushijima, T., Hosoya, Y., Ochiai, M., Kushida, H., Wakabayashi, K., Suzuki, T., Hayashi, M., Sofuni, T., Sugimura, T. and Nagao, M. (1994) Tissue-Specific Mutational Spectra of 2-Amino-3, 4-Dimethylimidazo[4, 5- $f$ ]Quinolone in the Liver and Bone Marrow of lacI Transgenic Mice. Carcinogenesis, 15, 2805-2809. https://doi.org/10.1093/carcin/15.12.2805

[24] Soultanakis, R.P., Melamede, R.J., Bespalov, I.A., Wallace, S.S., Beckman, K.B., Ames, B.N., Taatjes, D.J. and Janssen-Heininger, Y.M. (2000) Fluorescence Detection of 8-Oxoguanine in Nuclear and Mitochondrial DNA of Cultured Cells Using a Recombinant Fab and Confocal Scanning Laser Microscopy. Free Radical Biology and Medicine, 28, 987-998. https://doi.org/10.1016/S0891-5849(00)00185-4

[25] Wang, Y., Schulte, B.A., LaRue, A.C., Ogawa, M. and Zhou, D. (2006) Total Body Irradiation Selectively Induces Murine Hematopoietic Stem Cell Senescence. Blood, 107, 358-366. https://doi.org/10.1182/blood-2005-04-1418

[26] Wang, Y., Liu, L., Pazhanisamy, S.K., Li, H. and Zhou, D. (2010) Total Body Irradiation Causes Residual Bone Marrow Injury by Induction of Persistent Oxidative Stress in Murine Hematopoietic Stem Cells. Free Radical Biology and Medicine, 48, 348-356. https://doi.org/10.1016/j.freeradbiomed.2009.11.005

[27] Meng, A., Wang, Y., Van Zant, G. and Zhou, D. (2003) Ionizing Radiation and Busulfan Induce Premature Senescence in Murine Bone Marrow Hematopoietic Cells. Cancer Research, 63, 5414-5419.

[28] Insinga, A., Cicalese, A., Faretta, M., Gallo, B., Albano, L., Ronzoni, S., Furia, L., Viale, A. and Pelicci, P.G. (2013) DNA Damage in Stem Cells Activates p21, Inhibits p53, and Induces Symmetric Self-Renewing Divisions. Proceedings of National Academy of Science of the United States of America, 110, 3931-3936. https://doi.org/10.1073/pnas.1213394110

[29] Shao, L., Wang, Y., Chang, J., Luo, Y., Meng, A. and Zhou, D. (2013) Hematopoietic Stem Cell Senescence and Cancer Thrapy-Induced Long-Term Bone Marrow Injury. Translational Cancer Research, 5, 397-411.

[30] Shao, L., Kuo, Y. and Zhou, D. (2014) Hematopoietic Stem Cell Injury Induced by Ionizing Radiation. Antioxidants and Redox Signaling, 20, 1447-1462. https://doi.org/10.1089/ars.2013.5635

[31] Chung, Y.J., Robert, C., Gough, S.M., Rassool, F.V. and Aplan, P.D. (2014) Oxidative Stress Leads to Increased Mutation Frequency in a Murine Model of Myelodysplastic Syndrome. Leukemia Research, 38, 95-102. https://doi.org/10.1016/j.leukres.2013.07.008

[32] Pazhanisamy, S.K., Li, H., Wang, Y., Batinic-Haberle, I. and Zhou, D. (2011) NADPH Oxidase Inhibition Attenuates Total Body Irradiation-Induced Haematopoietic Genomic Instability. Mutagenesis, 26, 431-435.

https://doi.org/10.1093/mutage/ger001

[33] Moehrle, B.M., Nattamai, K., Brown, A., Florian, M.C., Ryan, M., Vogel, M., Bliederhaeuser, C., Soller, K., Prows, D.R., Abdollahi, A., Schleimer, D., Walter, D., Milson, M.D., Stambrook, P., Porteus, M. and Geiger, H. (2015) Stem Cell-Specific Mechanisms Ensure Genomic Fidelity within HSCs and upon Aging of HSCs. Cell Reports, 13, 2412-2424. https://doi.org/10.1016/j.celrep.2015.11.030 